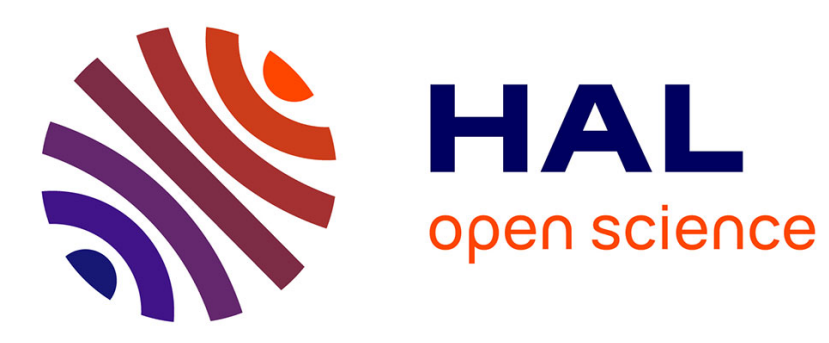

\title{
Deducing Altshuller's Laws of Evolution of Technical Systems
}

Justus Schollmeyer, Viesturs Tamuzs

\section{To cite this version:}

Justus Schollmeyer, Viesturs Tamuzs. Deducing Altshuller's Laws of Evolution of Technical Systems. 19th International TRIZ Future Conference (TFC), Oct 2019, Marrakesh, Morocco. pp.55-69, 10.1007/978-3-030-32497-1_6 . hal-02905526

\section{HAL Id: hal-02905526 \\ https://hal.inria.fr/hal-02905526}

Submitted on 23 Jul 2020

HAL is a multi-disciplinary open access archive for the deposit and dissemination of scientific research documents, whether they are published or not. The documents may come from teaching and research institutions in France or abroad, or from public or private research centers.
L'archive ouverte pluridisciplinaire HAL, est destinée au dépôt et à la diffusion de documents scientifiques de niveau recherche, publiés ou non, émanant des établissements d'enseignement et de recherche français ou étrangers, des laboratoires publics ou privés. 


\title{
Deducing Altshuller's Laws of Evolution of Technical Systems
}

\author{
Justus Schollmeyer ${ }^{1}$ and Viesturs Tamuzs ${ }^{2}$ \\ ${ }^{1}$ Second Negation, Berlin, Germany, justus@secondnegation.com 凶 \\ 2 Altshuller Institute, Worcester, Massachusetts, USA, viesturs.tamuzs@gmail.com凶
}

\begin{abstract}
According to Genrich Altshuller and most of his students, his eight laws of technical systems evolution were found by systematic patent analysis. In this paper, we show that not a single patent must be studied to derive the very same eight laws, but rather that they follow from an analysis of the concept of artificial instrumental systems in the context of a competitive world with scarce resources. Our investigation yields a clear definition of ideality which - insofar as certain constraints are respected - indicates multiple trends underlying the evolution of systems towards increased competitiveness when resources are scarce. By making the truth conditions for each law explicit, we attempt to explain why some high generality patents from the $20^{\text {th }}$ century contradict some of Altshuller's laws. We conclude that although patents can be seen as a useful source for inspiration, validation and falsification of generalizations, they are not the most promising place to start to look for laws of system evolution, since mere induction from past examples cannot provide the justification needed to meaningfully inform engineers about how to develop systems in the future. Our approach suggest an alternative route for articulating and justifying such laws.
\end{abstract}

Keywords: Laws of system development, Technological Forecast, Ideality.

\section{Goal and Structure of the Paper}

In 1979, Genrich Altshuller published 8 laws of technical systems evolution [1], which according to him [2] can be found by systematic study of high generality patents. Both, critics [3] and advocates [4] of the usefulness of Altshuller's laws emphasize that thousands of patents were analyzed to discover these laws. Although there is an abundant literature proposing divergent formulations of these and other laws that are often called trends or patterns (see, for example, [5-10]), we will concentrate on Altshuller's initial formulation from 1979 [1].

Our paper aims to show that, in principle, not a single patent must be studied in order to arrive at Altshuller's eight laws of technical system evolution. Rather, they follow from the meaning of a technical system when understood as an artificial instrumental system in the context of a competitive world with scarce resources. This deductibility is the actual reason why they might be able to lay claim to some law-like 
status $^{1}$ - at least as long as the respective conditions for their application are respected. This, however, does not imply that they should not be tested against the evidence from the patent stock (see section 4).

The paper comprises three major parts. First, we will articulate in general terms what systems are by putting our focus on the notion of instrumental systems, which can either be static or dynamic, and natural or artificial (section 2). Second, we will show that Altshuller's eight laws from 1979 can be inferred from the general statements explaining these distinctions - at least insofar as additional restrictions such as scarcity of resources and competition are assumed (section 3). Third, we will reflect on the counterexamples from a patent analysis done by [20] and show how the derived truth conditions for Altshuller's laws help explain these contradictions (section 4). Finally, we will summarize our findings and indicate how to proceed further (conclusions 5).

\section{General Reflection on Systems}

\subsection{Systems in General and Instrumental Systems in Particular}

A system is a whole that consists of parts, which - insofar as the system is not an abstract one - can be anything that has some mass. Together these parts bring about effects. Systems are useful as long as they can be controlled such that their ability to realize effects makes it possible to employ them for particular purposes in particular contexts. When systems are employed for particular purposes, they are serving functions and will be called instrumental.

\footnotetext{
${ }^{1}$ Traditionally, the term law requires some form of deductibility. According to [11], the debate about the definition of natural laws falls into two camps: systemic approaches and universal approaches. In the case of systemic approaches, natural laws are viewed in the context of deductive systems. Deductive systems consist of axioms and their logical consequences: theorems. Some of them are stronger than others, which means they are able to explain more. Some others are simpler and thus less complex. Simplicity and strength are seen as competing virtues insofar as a system can be made simpler by reducing strength, which can be achieved easily by elimination of some of the axioms resulting in a simpler system (see [11]). As a proponent of the systems approach, [12] believes that an account of our natural laws can be found in the totality of our true deductive systems that have the best strength-simplicity ratio. In contrast, universal approaches think about natural laws in terms of particular things being seen as instantiations of their universals. For example, a law that states that horses are mammals would assume the existence of (i) the universals horse-ness and mammal-ness and (ii) a necessitating relation - i.e. a relation of causality that is not merely logical [13] — between horse-ness and mammal-ness, such that horse-ness necessitates mammal-ness. Making such necessitating relations explicit in the form of laws allows for deduction. In both types of approaches - systemic and universal ones - deductibility is crucial for calling something a law. The way in which Altshuller's laws are usually presented in the literature - i.e. as being found by comprehensive patent analysis - rather resembles the style of universal approaches. In contrast, we are going to show that they follow from a handful of axioms within a simple deductive system.
} 


\subsection{Living Systems with Needs and Desires}

Living systems depend on instrumentalizing their environments. They must build cellular components from chemicals and energy (anabolism) and break down organic matter (catabolism) to either release energy or to serve anabolic reactions. These processes of metabolism require living systems to make use of what their environments provide. We will call a lack that would threaten a living system's healthy existence if not satisfied - a need. In addition to needs, some living systems - in particular humans - have desires. They come as the feeling of wanting.

\subsection{Satisfaction of Needs and Desires Requires Activity}

In order to fulfill a desire or to satisfy some need, living systems must do something. Humans, for example, must breathe to enrich themselves with oxygen and plants must convert sunlight into chemical energy for growing. While - for most people breathing is a subconscious activity most of the time, putting ourselves under water changes the situation entirely. Our standard technique of enriching our blood with oxygen will not work anymore.

\subsection{Solving Problems with Techniques and Technical Devices}

Living systems face problems, as soon as they are unable to fulfill some desire or to satisfy some need. Problems can be solved in a variety of ways. What can count as the most appropriate solution depends on context. Sometimes simple techniques such as breath retention can do the job. Sometimes, a basic technique is not enough, and some external enhancement is needed, for example a snorkel that allows us to breathe under water as long as the opening at the top of the snorkel remains above the waterline. In the latter case, we have employed something to help us satisfy our need to enrich our blood with oxygen by enabling us to breathe. We are not only using some technique but also some technical device.

\subsection{Artificial and Natural Systems}

While strolling around, we could be lucky and find some specially grown hollow wooden tree limb and use it is as a snorkel. Alternatively, we could make one on our own. In the first case, we would have to be able to recognize the function that the wooden limb could perform for us. In the second case, we will have to purposefully process something such that it can serve this function. In both cases, we must be able to recognize our need and understand that we are looking for something that makes it possible for us to breathe under water. It might already exist and we have to find it, or it might not exist yet and we must create it. We will call everything that was purpose- 
fully created or manufactured artificial to distinguish it from natural things that were not purposefully put together by someone. ${ }^{2}$

\subsection{Functional Parts}

In its simplest version, a snorkel is a hollow stick that is U-shaped at the bottom. The shorter leg fits into a human's mouth, while the longer leg is straight and reaches out of the water when used as a snorkel. Although the device consists of only one part, it can be called a system, because from a functional perspective, multiple parts can be distinguished: the "mouth part", the "channel part", and the "chimney part". Technically, functions are expressed in the form "part A + function (verb + measurable noun) + part B". Take, for example, the channel part (A), which constrains (verb) the movement (measurable noun) of the air flow (molecules [B]) within the snorkel.

\subsection{Energy Transformation and Static Systems vs. Dynamic Systems}

A function that can be executed while an equilibrium is reached such that the responsible parts do not have to expend any energy - as, for example, in "the screw positions the plate" - will be called static. ${ }^{3}$ In contrast, functions will be called dynamic if their execution requires any of the responsible parts to expend energy, as in "the lever changes the position of the basket" (where something or someone needs to move the lever). Accordingly, we will speak of a static system when taking into account static functions alone - for example when reflecting on how the snorkel is constructed. Analogously, when taking into account dynamic functions as well, we will call the system dynamic. Since energy cannot be produced from nothing, dynamic systems have to be their own energy sources by somehow transforming energy from their environments. Energy transformation is thus a key feature of dynamic systems.

\subsection{The Functions of Systems Show in the Respective Dynamic Super-Systems}

Due to their dependence on dynamic systems, we would not be able to identify a static system's function if we were not able to imagine or observe it performing its roles within dynamic super-systems. ${ }^{4}$ For example, a bicycle on its own is a static system.

\footnotetext{
2 The totality of roles that an artificial system can be employed for does not have to be equal to the set of roles it was designed for in the first place. A bridge, for example, can be designed for allowing cars to cross a river, but it might also provide shelter from rain.

3 Note that this notion of a static function is an abstraction, since, upon closer consideration,
functions can only be called static when abstracting from undesired dynamics - for example
material wear in the screw that positions a plate.

${ }^{4}$ Note that some of the functions responsible for keeping the static system's form can be identified irrespective of the system's role in dynamic super-systems.
} 
When being ridden, it is part of a dynamic super-system within which it serves a function. A bike has the disposition to become dynamic, but it is not dynamic in itself. The same holds for the snorkel. Only when used by someone for snorkeling does this static system become part of a dynamic super-system and show its proper function. In one sentence: to understand functions of systems, we have to investigate them in the context of the dynamic super-systems where they satisfy some need or desire.

\subsection{Hierarchical System Structure}

Dynamic super-systems can themselves be systems in some wider dynamic supersystem. A cyclist on a treadmill, for example, can be part of an energy generating fitness center. The treadmill (static system) and the cyclist (dynamic system) form a dynamic super-system, which is part of the larger dynamic super-super-system that consists of multiple ridden treadmills, a battery etc. Most of the time, systems exist in much more complex hierarchies than this one and it would be pointless to add the prefix "super" to each higher or "sub" to each lower level. From now on, we will therefore abandon this practice if no additional clarity can be gained by pointing out the different levels of hierarchy.

\subsection{Energy Input as a Key Feature of Dynamic Systems}

Although the ridden treadmill transforms mechanical energy into electrical energy, it is a static system because it requires a cyclist to receive energy in the first place. Therefore, the ability to transform energy is not enough for a system to be dynamic. Suppose the cyclist was replaced by a combustion engine providing the mechanical energy needed to turn the shaft of the pedals. As long as they are being fueled, engine (dynamic) and treadmill (static) would become a dynamic system. Energy input is thus a further key feature of dynamic systems. A dynamic system that is cut off from its energy source will sooner or later turn into a static system.

\subsection{Dependence versus Relative Independence}

If a dynamic system does not have control over its own energy input, it is dependent on the dynamic system controlling it. This dependency comes in different forms. Electronic devices have power switches that are controlled by their users, solar thermal heating systems depend on whether or not the sun is shining and mammals have to find food or water to fuel their metabolism. Roughly speaking, the dynamic system controlling another's energy input determines whether or not the latter will remain dynamic or become static. Generally speaking, the more control a dynamic system gains over its fundamental operational functions such as energy input, the more independent it becomes. 


\subsection{Energy Conduction, Control, and Work as Key Features of Dynamic Sys- tems}

As far as we know, energy can neither be destroyed nor created, but only transformed from one form to another. That dynamic systems transform energy shows in their production of effects. Some of these effects might be useful for someone while others might not. This usefulness of the produced effects is the result of their work and it is also the reason why they are employed by others for their purposes. However, the work a dynamic system is employed for is different from the work carried out by the dynamic system's mechanism. When we want to turn a shaft by means of a combustion engine, the engine must burn fuel to transform chemical energy into mechanical energy. Besides guiding the input energy to its place of conversion and transforming it, the energy must be directed to where it can do the work it is supposed to do. Directing energy requires both control over the energy flow and the capacity to conduct energy from input, via transformation, to output. Figure 1 contains a summary of what we can say about dynamic systems in general terms.

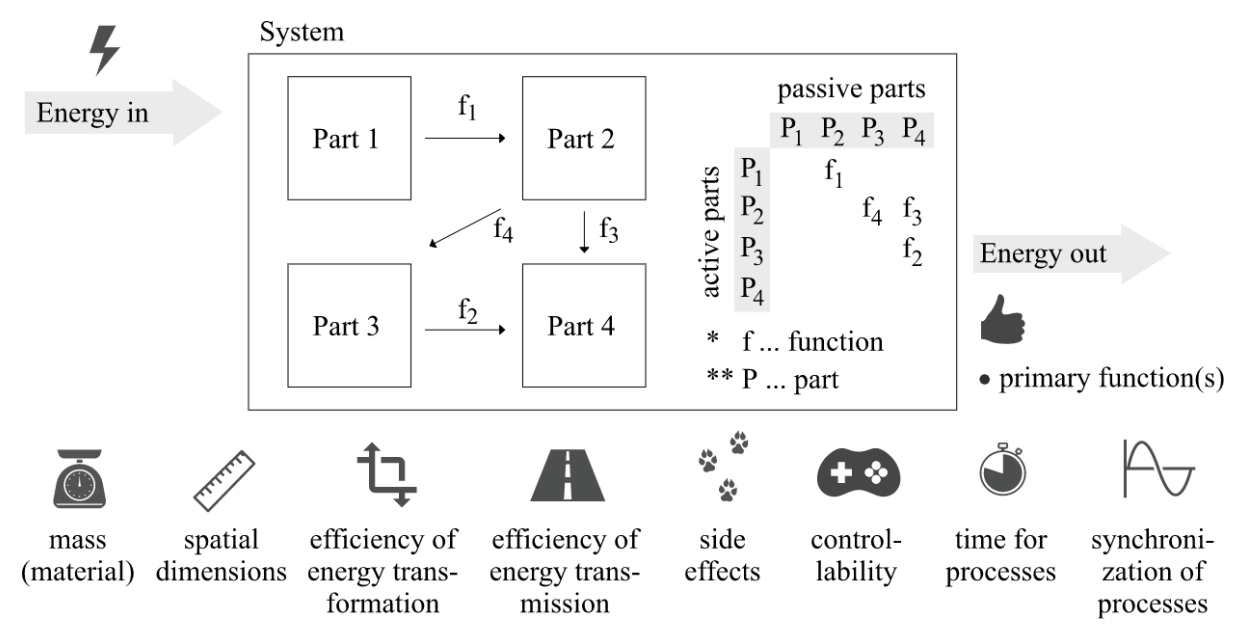

Fig. 1. The illustration captures necessary features of (instrumental) dynamic systems: They consist of parts which together bring about effects by transforming and directing energy such that it becomes useful for someone's purposes - primary function(s). The interactions of the parts can be described as processes that require a certain amount of time to be executed and that must be synchronized in order to work together under sufficiently controlled circumstances. The parts themselves have a mass and spatial dimensions. Insofar as we know the ratio between the amount of energy being consumed and being outputted in a useful manner, both energy transformation and transmission efficiency can be determined. Efficiencies lower than $100 \%$ result in side effects - i.e. in effects different from the system's primary function(s). 


\subsection{Scarcity of Resources and Efficiency}

Those who employ a static or dynamic system for their own purposes are interested in the output produced by the dynamic super-system emerging during this process. The output shows in the work being done. This work comes at some cost. It is tempting to say that a dynamic super-system that consumes less energy to produce the same amount of work under similar conditions and with more or less the same undesired side effects is better than a system that consumes more. However this only holds if the energy resource we are talking about is scarce or at least hard to obtain. Assuming such scarcity, efficiency gains become desirable.

Departing from the above analysis of general features of dynamic systems (see Fig. 1), we can compare the efficiencies of systems serving the same primary functions by relating these functions to the invested energy (see equation (1)). This energy comprises (i) the energy being lost during operation, (ii) the energy contained in the useful work of the primary function(s), (iii) the energy required for manufacturing and (iv) the energy stored in the form of materials (mass). In order to increase a system's overall efficiency - which is desirable under the assumption that resources are scarce, we will have to choose one or multiple of the options expressed within the following equation:

efficiency $\uparrow=\frac{\text { primary function } \uparrow}{\text { energ } y_{\text {invested }} \downarrow}=\frac{\text { primary function } \uparrow}{\text { energ } y_{\text {lost }} \downarrow+e n e r g y_{\text {out }} \downarrow+e n e r g y_{\text {manuf }} \downarrow+e n e r g y_{\text {stored }} \downarrow}$

\subsection{From Efficiency to Ideality}

While scarcity of resources makes efficiency gains desirable, it can be said that the pressure of competition forces systems to evolve towards being more efficient. Pushing the tendencies for increasing efficiency to their most extreme values (zero and max) or - as Altshuller would say - to their ideal forms, we will obtain Altshuller's famous definition of ideality (where no resources are needed to obtain the benefit of the system's function): The ideal system is the fulfillment of the function without a system [14]. Figure 2 summarizes these tendencies - and thereby the meaning of ideality - in one table. As a consequence, it can be said that a system that develops according to these tendencies is becoming increasingly ideal:

5 An upward arrow stands for increase and a downward arrow for decrease. The upward arrow behind primary function indicates both quantitative and qualitative improvement, while quantitative improvement means larger amount of primary functions. 


\begin{tabular}{|c|c|c|c|c|c|}
\hline Feature & Ideal value & Feature & Ideal value & Feature & Ideal value \\
\hline $\begin{array}{l}\text { energy in } \\
\text { energy out }\end{array}$ & & $\begin{array}{l}\text { efficiency of } \\
\text { energy trans- } \\
\text { formation }\end{array}$ & $\sqrt{4}$ & $\begin{array}{l}\text { control- } \\
\text { lability }\end{array}$ & $\frac{1}{4}$ \\
\hline $\begin{array}{l}\text { primary } \\
\text { function(s) }\end{array}$ & & $\begin{array}{l}\text { efficiency of } \\
\text { energy trans- } \\
\text { mission }\end{array}$ & is & $\begin{array}{l}\text { time for } \\
\text { processes }\end{array}$ & $v^{\prime}$ \\
\hline $\begin{array}{l}\text { mass } \\
\text { (material) }\end{array}$ & & $\begin{array}{l}\text { side } \\
\text { effects }\end{array}$ & w & $\begin{array}{l}A- \\
\text { synchroni- } \\
\text { zation of } \\
\text { processes }\end{array}$ & $\frac{1}{4}$ \\
\hline $\begin{array}{l}\text { spatial } \\
\text { dimensions }\end{array}$ & & & & & \\
\hline
\end{tabular}

Fig. 2. The illustration shows the extreme values for each of the general features of dynamic systems, indicating the respective tendencies for an increase of the system's overall efficiency. Accordingly, both the energy put in and the energy (work) required to fulfill the primary function(s) should be zero as should be the parts' masses, spatial dimensions (when space is considered a scarce resource), side effects and execution time. In contrast, the amount and quality of the primary function(s) should be maximal, as should be the energy transformation and transmission efficiencies, overall controllability and synchronization of processes.

\subsection{Integration in Wider Super-Systems}

From the tendency to become increasingly ideal, a trend toward functional integration into larger super-systems can be inferred: ${ }^{6}$ (i) A system that is serving multiple functions is more ideal than (ii) multiple systems that are serving all of these functions individually, assuming that (i) the multi-functional system consumes fewer resources (space, time, energy, materials) and (ii) that the quality of the execution of the functions does not suffer from the integration.

\subsection{Integration Means Mediating Conflicts}

Functional integration yields conflicts insofar as systems that are to be integrated compete over shared resources (space, time, material resources, energy). Systems that are able to integrate their sub-systems in a win-win fashion will be more efficient and therefore more competitive and more ideal than those that cap the potential of their individual sub-systems due to inner-systemic conflicts.

${ }^{6}$ See Fig. 2 "primary function(s)" (MAX). 


\subsection{Increase of Efficiency through Increase of Control over More Fundamental Activities}

When taking a look at the energy flow in dynamic systems above, we came across (i) energy input, (ii) energy transformation, (iii) energy conduction, (iv) energy control and (v) targeted energy output as necessary functions. The efficiency of each of these functions contributes to the system's overall efficiency. Loss of energy during any of these activities diminishes the system's overall efficiency and therefore provides opportunities for increasing ideality through problem solving. ${ }^{7}$ Since energy cannot get lost, loss of energy refers to transformation of energy into a form that cannot be used for executing the system's functions and, in this sense, is wasted. The better a dynamic system controls the activities underlying these functions, the more it will get out from its initial energy input. 8

The idea behind this principle is simple: intervening closer to where an activity originates allows for more controlled guidance of resulting effects as compared to intervention on the level of later emerging effects. For example, a central bank that aims at increasing investments into the economy by lowering interest rates cannot determine what banks will do with the cheap money. In contrast, direct control of the decision making of the banks would allow for more targeted intervention. The same holds for effects emerging from the activities of objects on an atomic and sub-atomic scale such as electromagnetism or material properties. The better we understand and control the origin of the effects of interest, the better we will control the effects themselves. Since increasing ideality is achieved by reducing losses while increasing functionality, systems that efficiently control the effects they exploit on the most fundamental level of their emergence, will turn out to be more ideal than systems operating

7 See Fig. 2 "efficiency of energy transmission" (MAX), "efficiency of energy transformation" (MAX), "energy in" (ZERO), "energy out" - corresponding to the work of the primary function (ZERO), “controllability” (MAX).

${ }^{8}$ How efficiently energy can be transformed by a given system depends on its ability to release the energy that is stored in what the system takes as input. Given that the maximal energy of a body is equivalent to its mass, a dynamic system can operate with increasing amounts of energy, the more control it gains over the matter it is dealing with. Burning a kilogram of dry straw provides approximately $16.8 \mathrm{MJ}$ in energy. The loss of rest mass of about $1.9 \times 10^{-7} \mathrm{~g}$ is so small that it is nearly impossible to measure. In contrast, when uranium decays, $0.1 \%$ of its mass is released as energy. After the reaction, the mass of the substance that initially was one kilogram of uranium is about one gram smaller, which is equivalent to a release of $9 \times 10^{7} \mathrm{MJ}$. To use uranium as an energy source, we must be able to trigger the required chain reactions and control them adequately, which requires understanding and control of the processes on an atomic scale. In contrast, in order to burn and control one kilogram of straw, all we have to know is how to initiate and sustain a fire, which can be done on a macro-scale level. If we were able to transform the entire kilogram of anything's rest mass into energy, we would obtain roughly $9 \mathrm{x}$ $10^{9} \mathrm{MJ}$. To do so we would have to be able to control the building blocks of matter on the lowest scale possible. 
at a later stage in the chain, where losses have already accumulated. If the origin of the effects exploited by some system resides at the sub-atomic scale, the system will have to obtain better control over the lower-scopic ${ }^{9}$ levels to become increasingly ideal. As more refined physical cause-effect relationships become known for further exploitation, we will see an increase in systems using them for their purposes. This holds for both the creation of artificial instrumental systems and for our understanding of the causal mechanisms operating in natural dynamic systems.

\section{Inferring Altshuller's Eight Laws of Technical Systems Devel- opment}

This general understanding of systems is sufficient to derive Altshuller's eight laws as stated in 1979 [1]. Altshuller split them into three groups: A) statics, B) kinematics, and C) dynamics. Note that Altshuller's usage of the terms "statics" and "dynamics" differs from our usage.

A) The first class of laws deals with the beginning of a technical system's life, which is understood as the result of synthesizing parts into a whole.

1) Law of system completeness: The first law states that a technical system would not be able to perform its functions, unless at least the following four parts with their respective functions are present: engine (for energy conversion), transmission, working unit and control unit. At least one of these parts must be manageable to make the system manageable.

As we have seen, this statement only holds for dynamic systems. Static systems, such as tables, picture frames, plates, chairs, unridden bicycles, etc. do not have to transform energy in any meaningful manner in order to provide their functions. ${ }^{10}$ The need for the five functions - (i) energy input, (ii) transformation, (iii) transmission, (iv) control and (v) targeted output (working unit in Altshuller's sense) follows from the fact that dynamic systems have to convert and control energy to bring about the effect that makes them instrumental for something (instrumental systems), themselves (selfsustaining living systems) or both (see 2.7, 2.10, and 2.12).

\footnotetext{
9 We avoid the term "microscopic" since "micro" has the well-defined meaning of 10-6. By coining the term lower-scopic, we want to convey the meaning of a spectrum for zooming out (macro-scale) and zooming in (towards sub-atomic scale).

10 When zooming onto an atomic and sub-atomic level, we will find dynamic energy conversion, irrespective of whether we are analyzing a static or a dynamic system. However, this does not affect the meaning of the distinction between static and dynamic on a macro-level.
} 
2) Law of energy conductivity: Altshuller's second law states that a system's need for energy transformation requires the ability to conduct energy from the conversion unit to the working body. As we have seen, this can only be said about dynamic not static systems and is already contained in the meaning of transmission/conduction (see 2.12).

3) Law of synchronization: The third law states that all parts of the system should work in coordinated rhythm.

Again, this only holds for dynamic systems and is due to the requirement that multiple parts have to contribute to the same process, which is the production of the system's effects through energy conversion and control. Insofar as the parts contribute to the same process their contribution has to be coordinated in time, which is just a different way of saying that coordination of rhythm is required.

B) Altshuller put the second set of laws under the title "kinematics" stating that they refer to the development of technical systems irrespective of specific technical or physical factors contributing to the development.

4) Law of increasing degree of ideality: Altshuller's fourth law is probably the most popular one and claims that all technical systems develop towards an increasing degree of ideality with the system's weight, volume and area tending towards zero, without diminishing its capacity to perform work, resulting in Altshuller's popular definition of an ideal technical system as function without a system [14]. In the TRIZ literature, it is common practice to understand ideality not only in terms of spatial and material resources, but also in terms of all resources (time, space, energy input, material resources) in the form of a qualitative cost-benefit equation with functionality above and costs and problems below the fraction line [15]. In [5], which is a translation of [16], ideality is defined as the ratio between useful and harmful effects. ${ }^{11}$

As seen above, the trend of increasing ideality (summarized in Fig. 2) is due to the need to increase efficiency (see 2.13 and 2.14). Increased efficiency, in return, provides a competitive advantage when resources are scarce. While the law of increasing ideality refers to both static and dynamic systems, it depends upon the assumption of competition and scarcity of resources. It would be an interesting thought experiment to investigate what would happen to the trend towards an increasing degree of ideality if the problem of scarcity of resources were solved.

11 According to [17], the translation contains slight modifications, but we currently have no access to [16] to judge whether or not they affect this definition of ideality. 
5) Law of uneven development of a system's parts: The fifth law states that the parts of a technical system develop unevenly. As a consequence, system conflicts arise, where one sub-system hinders the further development of another. Altshuller's example here is the insufficiency of brake systems in large ships [18].

This argument depends on two assumptions. The first is the system definition itself stating that systems bring about their effects by collaborative work of their parts. Due to the dependency of systemic effects on the interaction of all of the relevant parts of a given system, weaker parts will be bottlenecks for the stronger ones or the system will become unstable when weaker parts cannot balance out the stronger. The second assumption, which is not contained in the system definition itself, is that these parts do not develop simultaneously. This might be true if the system's designer was unilaterally focused. However, the law will collapse as soon as systems are developed from a holistic perspective by taking the functions of all sub-systems into account.

As we have seen above, there is another argument for the necessity of the outbreak of system conflicts that does not rely on assuming a limited perspective of the designer (see 2.16). Rather, it is based on resource dependency. Different sub-systems might exploit shared resources (energy, material, time and space), which will result in conflicts when these resources are scarce.

6) Law of transition to the super-system level: Altshuller's sixth law states that when systems have exhausted their developmental potential, they become sub-systems within larger systems - that is their function is integrated into a super-system. As we have seen, this follows from the ideality statement under the condition that the function of the integrated systems is enhanced rather than worsened and that the non-integrated individual systems would require more resources for delivering their function (see 2.15).

C) The third class of Altshuller's laws is titled "dynamics" and reflects the main trends in the development of technical systems in our - or rather Altshuller's - time; Creativity as an Exact Science was published in 1979 [1].

7) Law of transition from the macro- to the micro-level: The seventh of Altshuller's laws claims that when a system's developmental potential is exhausted on the macro level, it will be fundamentally rebuilt with its working body acting on a lower-scopic level. As we have seen, this is due to the principle that the ability to control the more fundamental activities from where the effects emerge provides more control over the entire process. Therefore, less energy loss is accumulated, which results in higher efficiency and thus a higher degree of ideality. Moreover, the production of new effects is made available (see 2.17). 
8) Law of increasing involvement of substance-field systems: The eighth of Altshuller's laws finally states that the development of technical systems proceeds towards an increasing involvement of substance-field systems. In order to clarify the meaning of this statement, we first need to look at the role that substance-field (SuField) systems play in Altshuller's theory. Roughly, the Su-Field notation aims at representing problems in terms of cause-effect relationships by means of three major categories: substances, fields and actions. Fields are supposed to act upon and to be produced by substances. The actions of being produced and acting upon something are represented by means of arrows, which can highlight qualitative differences (harmful, useful, insufficient, and excessive). This notation is used to represent problems and can be converted into the notation of functional modeling where components (substances) act via functions (arrows) upon other components. The main difference between function and field seems to concern the level of abstraction. While fields are categories for physical effects, functions describe the activity by which one component acts upon another. In order to preserve the information represented in a Su-Field model which states that "A pushes B" by means of a mechanical field, we would have to say "A mechanically pushes B". Thus, in a Su-Field representation, the physical principles bringing about some effect are made explicit (something that is not necessarily shown in a functional diagram, but could easily be added).

Altshuller's eighth law states that no-field systems tend to develop into field systems (note that Altshuller's notion of a no-field system might be equivalent to what we are calling static system $)^{12}$, the number of connections between the elements of a system increases, the responsiveness of the system increases, and mechanical mechanisms are replaced by electromagnetic ones.

All of these observations result from statements outlined before, namely that (i) the ability to efficiently control lower-scopic effects often makes it possible to influence matter more efficiently and extensively than handling them on a macroscopic mechanical scale (see 2.17) and that (ii) static systems are only useful insofar as they are integrated into dynamic super-systems (which refers to no-field systems turning into SuField systems) (see 2.7 and 2.8). Note that (i) is only true if we can control the lowerscopic behavior efficiently enough to gain an advantage over macroscopic mechanical control.

12 Take for example a hammer, a nail and a wall. Without what Altshuller would call a mechanical field that is provided by, for example, someone's arm, the nail could not be hammered into the wall. According to our terminology, we need a dynamic system (the person who takes and controls the hammer) to turn the static systems - hammer, nail, wall — into a dynamic supersystem: The person uses the hammer to hammer the nail into the wall. 


\section{Patent Analysis for Validation/Falsification - not for Induction}

We have shown that, without the study of a single patent, Altshuller's eight laws of technical system development from 1979 [1] can be derived from a reflection on the nature of systems, in general, and instrumental systems in particular. All in all, this shows that the law-like nature of these general statements about systems is due to their inferability from even more general statements. Instead of trying to derive laws of technical system evolution from patent analysis, it seems to be more promising to use the patent fund as resource for validation. In 2006, [20] tested Altshuller's laws in the following manner. From [21] they took the 68 highest generality US patents from 1963-1999, which are patents that are cited by subsequent patents belonging to a wide range of International Patent Classification (IPC) classes. For each of these patents, the authors studied the state of the art section in order to understand the transition from the state of the art situation to the solution in the respective patent. For each of the technical system evolution trends, they checked whether or not it could have been used for developing the solution and whether or not it contradicted the transition proposed in the patent. Altogether, the 68 patents provided examples for each of Altshuller's laws, while 3 patents provided counter-examples concerning law 2) energy conductivity, law 5) uneven development of a system's parts, and law 8) increasing use of su-fields. Despite our inability to get in touch with the authors of [20] to gain access to supplementary materials to view the counter-examples, our above analysis has shown that the contradicted laws are conditional, which might explain the contradictions: Law 2 does not hold for static systems, law 5 is not valid when the product was developed from a holistic standpoint, and law 8 depends on efficiency concerns, which allows for the possibility that a mechanical solution is more efficient than its non-mechanical predecessor.

\section{Conclusion}

As we have shown, Altshuller's eight laws of technical system evolution can be derived from a general reflection on artificial instrumental systems in the context of a competitive world with scarce resources. As a result, the conditions for applying the laws could be made explicit, which helped to hypothesize why 3 out of 68 high generality patents contradicted 3 out of 8 laws in the study of [20] (see section 4). While patents are a useful source for inspiration, validation and falsification of generalizations, they are not the most promising place to start to look for laws of system evolution since mere induction from past examples cannot provide the justification needed to meaningfully inform engineers about how to develop systems in the future. Moreover, we drew the attention to resource scarcity as a fundamental condition for the validity of most of these laws, which allowed us to summarize the meaning of ideality in a deductive manner (see Fig. 2). Given that the number of general resource types is 
limited - time, space, material, energy - we believe that additional general statements about systems can be derived by further taking into account the general characteristics of each resource type in their respective contexts. Doing so might result in the formulation of trends similar to the patterns articulated in [19]. The same holds for (i) a more thorough investigation of the fundamental features of dynamic systems (see Fig. 1 and Fig. 2), (ii) a closer look at differences of employing living or non-living systems for certain jobs, and (iii) a definition by cases depending on whether the instrumental system is abstract (theories) or concrete (material).

\section{References}

[1] Altshuller, G.: Creativity as an Exact Science: The Theory of the Solution of Inventive Problems. Studies in Cybernetics : A Series of Books and Monographs Covering All Aspects of Cybernetics. - New York, NY : Gordon \& Breach, 1982-1994 5. New York, NY [u.a.]: Gordon and Breach, 1984, 223-231.

[2] Ibid., 30-36.

[3] Filkovsky, G.L.: "Why Laws of Technical Systems Evolution Can’t Successfully Predict a System Evolution.” Anti TRIZ-Journal 3, no. 1 (2004). http://www3.sympatico.ca/karasik/GF_why_LOTSE_cannot_predict_system_evolution.html.

[4] For example, Fey, V., and E. Rivin: "Innovation on Demand by Victor Fey." Cambridge Core, October 2005. https://doi.org/10.1017/CBO9780511584237, 113.

[5] Altshuller, A., B. Zlotin, A. Zusman, and V. Philatov.: Tools of Classical TRIZ. Southfield, Mich: Ideation Intl Inc, 1999.

[6] Savransky, S. D.: Engineering of Creativity: Introduction to TRIZ Methodology of Inventive Problem Solving. CRC Press, 2000.

[7] Petrov, V.: "The Laws of System Evolution.” The Triz Journal (blog), 2002. https://triz-journal.com/laws-system-evolution/, 2002.

[8] Mann, D.: Hands on Systematic Innovation: For Business and Management.

Clevedon: Edward Gaskell Publishers, 2004. 
[9] Fey, V., and E. Rivin: "Innovation on Demand by Victor Fey." Cambridge Core, October 2005. https://doi.org/10.1017/CBO9780511584237.

[10] Bukhman, I.: TRIZ Technology for Innovation. Taipei: Cubic Creativity Company, 2012.

[11] Carroll, John W.: "Laws of Nature." In The Stanford Encyclopedia of Philosophy. Edward N. Zalta, 2016. https://plato.stanford.edu/archives/fall2016/entries/laws-ofnature/.

[12] Lewis, David K. Counterfactuals. Blackwell, 1973, 73.

[13] Armstrong, D. M.: "The Identification Problem and the Inference Problem." Philosophy and Phenomenological Research 53, no. 2 (1993): 421-422.

[14] Altshuller, G.: Creativity as an Exact Science: The Theory of the Solution of Inventive Problems. Studies in Cybernetics : A Series of Books and Monographs Covering All Aspects of Cybernetics. - New York, NY : Gordon \& Breach, 1982-1994 5. New York, NY [u.a.]: Gordon and Breach, 1984, 223-231.

[15] Fey, V., and E. Rivin. "Innovation on Demand by Victor Fey." Cambridge Core, October 2005. https://doi.org/10.1017/CBO9780511584237, 17.

[16] Altshuller, G., B. Zlotin, A. Zusman, and V. Philatov. Search for New Ideas: from Insight to Technology (Theory and Practice of Inventive Problem Solving). Kishinyov: Cartia moldovenyaske, 1989.

[17] Domb, E.: "Book Review: Tools of Classical TRIZ." The Triz Journal (blog), 1999. https://triz-journal.com/book-review-tools-classical-triz/.

[18] Altshuller, G.: Creativity as an Exact Science: The Theory of the Solution of Inventive Problems. Studies in Cybernetics : A Series of Books and Monographs Cover- 
ing All Aspects of Cybernetics. - New York, NY : Gordon \& Breach, 1982-1994 5. New York, NY [u.a.]: Gordon and Breach, 1984, 229.

[19] Altshuller, A., B. Zlotin, A. Zusman, and V. Philatov. Tools of Classical TRIZ. Southfield, Mich: Ideation Intl Inc, 1999, 11-23.

[20] Carvalho, M. A. De, R. L. V. D. Rocha, A. P. Zanoni, H. F. Boell, N. Back, and A. Ogliari. "Validity of Technical System Evolution Trends - a Patent Study." Anais Do I Congresso Iberoamericano de Inovação Tecnológica. Puebla, México: Asociación Mexicana de TRIZ (AMETRIZ), 2006.

[21] Hall, B. H., A. B. Jaffe, and M. Trajtenberg. "The NBER Patent Citations Data File: Lessons, Insights and Methodological Tools," 2001. https://www.nber.org/papers/w8498.pdf. 